\title{
Reproductive behaviour of the black-striped pipefish Syngnathus abaster (Pisces; Syngnathidae)
}

\author{
K. Silva*†t, N. M. Monteiro $†$, M. N. Vieira*† \\ And V. C. Almada \\ *Departamento de Zoologia e Antropologia da Faculdade de Ciências da Universidade \\ do Porto, Praça Gomes Teixeira, 4099-002 Porto, Portugal, †CIIMAR, Rua dos Bragas \\ 177, 4050-123 Porto, Portugal, §Faculdade de Ciências da Saúde da Universidade \\ Fernando Pessoa, Rua Carlos da Maia 296, 4200-150 Porto, Portugal and \\ ||ISPA, Rua Jardim do Tabaco 34, 1149-041 Lisboa, Portugal
}

(Received 25 July 2005, Accepted 3 July 2006)

\begin{abstract}
The reproductive behaviour of Syngnathus abaster is described and compared with those of other syngnathids. The need for standardized behavioural data is discussed in light of the actual theories of evolution of mating patterns and sex-role reversal within this family.

(C) 2006 The Authors Journal compilation $(2006$ The Fisheries Society of the British Isles
\end{abstract}

Key words: courtship; reproduction; sex-role reversal; Syngnathidae; Syngnathus abaster.

The Syngnathidae (comprising pipefishes, seahorses and seadragons) is well known for its unique form of parental care, where females deposit unfertilized eggs into the male's specialized ventral incubating surface (Herald, 1959). Together with male pregnancy, some syngnathid species also exhibit varying degrees of sex-role reversal (Monteiro et al., 2002; Berglund \& Rosenqvist, 2003), with females competing for access to mates and sometimes presenting conspicuous secondary sexual characters. These specializations have promoted the use of syngnathids as models in which to study not only the evolution of parental care (Trivers, 1972; Ridley, 1978), but also to test theoretical assumptions of sexual selection (Johnstone, 1995).

The phylogenetic relationships among syngnathids have been studied both by Herald (1959), based on the morphological organization of the male's incubating area, and Wilson et al. (2003), using mitochondrial DNA, producing similar results. Attempts to determine the evolution of mating patterns or the degree of sex-role reversal, superimposing information from behavioural and ecological data are, nevertheless, still far from producing definitive results. Despite the

\#Author to whom the correspondence should be addressed. Tel.: +351 22 3401521; fax: +351 22 3401511; email: ksilva@fc.up.pt 
ubiquity of male pregnancy in syngnathids, mating patterns and sexual dimorphism differ greatly among species. Thus, comparative studies must be preceded and supported by objective and reasonably standardized behavioural observations, able to highlight not only differences between species but also variation among populations.

In order to further extend knowledge of syngnathid reproduction, a study area where information on the most basic life-history parameters is scarce, data were collected on the reproductive biology of the black-striped pipefish Syngnathus abaster Risso, based on aquarium observations of individuals captured in the wild. The reproductive behaviour of this species is poorly understood and information on its reproductive biology is still insufficient. Tomasini et al. (1991) described factors that influence the reproductive success of a population of $S$. abaster in a Mediterranean lagoon (Mauguio, France), Carcupino et al. (1997) described the ultrastructural organization of the male brood pouch epithelium and Silva et al. (2006) presented a description of the embryonic development and juvenile behaviour. Furthermore, comparisons are presented and discussed, highlighting similarities and differences in the reproductive biology of the black-striped pipefish when compared to other Western European pipefish species.

Syngnathus abaster, a euryhaline species with a restricted distribution that includes the Mediterranean and Black Sea northward to southern Biscay (Dawson, 1986), occurs either in coastal areas or in brackish and fresh waters (Cakic et al., 2002). The black-striped pipefish is a small brown-green pipefish, with dark or pale spots on the trunk and tail. It can be found mainly among sand, mud or eelgrass meadows, between depths of 0.5 and $5 \mathrm{~m}$, within a temperature range of 8 and $24^{\circ} \mathrm{C}$. Males of the Urophori have a brood pouch located ventrally on the tail, which consists of two skin folds that contact medially with their free edges.

Fish were collected with a hand-net, in a salt pond reservoir, at the Ria de Aveiro estuarine lagoon $\left(40^{\circ} 45^{\prime} \mathrm{N} ; 8^{\circ} 40^{\prime} \mathrm{W}\right)$, Portugal. One hundred and seventy-six mature individuals, including males and females, were captured during a 2 year period (March 2003 to June 2005), measured for total length $\left(L_{\mathrm{T}}\right)$ and the number of eggs (visible through the marsupium folds) was counted in each pregnant male. These individuals were transported to the laboratory and maintained in several 801 aquaria, illuminated with natural light and supplemented with $18 \mathrm{~W}$ fluorescent lamps. The tank substrata consisted mainly of sand and plastic seagrass laid in order to mimic the original habitat where the fish were caught. Due to the 'gas bubble disease', common in pipefishes, aeration was performed outside the fish tanks (Monteiro et al., 2002). The continuously running sea water was physically and biologically filtered and its temperature and salinity (33) maintained constant. Fish were fed daily with fresh Artemia franciscana nauplii. Adult males and females were initially kept separate in order to synchronize the 'disposition to mate' and simultaneously to allow the fish to be accustomed to the presence of an observer. An equal number of females and non-brooding males (not exceeding six individuals) were then placed together to mate. Since no successful mating rituals were observed in the first courtship trials, with the water temperature ranging from 14 to $15^{\circ} \mathrm{C}$, and considering that temperature is one of the strongest factors in the control 
of marine fish reproduction (Monteiro et al., 2001), water temperature was gradually increased to $18-19^{\circ} \mathrm{C}$. This is in agreement with in situ observations (during 2005, adult $S$. abaster migrated to salt ponds, in order to reproduce, when temperature reached values $>18^{\circ} \mathrm{C}$; K. Silva, pers. obs.). This adjustment in water temperature resulted in the observation of several successful matings. More than $230 \mathrm{~h}$ of ad libitum observations were conducted (Martin \& Bateson, 1993), at random intervals, and the main stages of the courtship rituals were defined, measured and described. More than $15 \mathrm{~h}$ of video-tape recordings were also used in order to further describe the behavioural patterns of courtship and mating.

Differences between male and female initiative, flickering movements and leads were determined using ADERSIML (Almada \& Oliveira, 1997). This computer programme implements a procedure to access the significance of goodness of fit tests that would usually be addressed using the $\chi^{2}$ distribution. This procedure was chosen because it allows the analysis of data where expected frequencies are very low $(<4$ or even 0$)$ in several classes, a condition in which the $\chi^{2}$ distribution must not be used (Zar, 1984).

Syngnathus abaster males and females differed significantly in $L_{\mathrm{T}}$ [t-test, $n=$ 176 (79 females and 97 males), $P<0.001]$ with females being larger than males (female: mean $=9.19 \mathrm{~cm}$, range: $6.7-12.7 \mathrm{~cm}$; male: mean $=8.46 \mathrm{~cm}$, range: $5 \cdot 2-13 \cdot 5 \mathrm{~cm})$.

Pregnant males caught in salt ponds carried an average of 37 eggs $(n=58$, mean \pm S.D. $=37 \pm 11$ eggs, range: $10-64$ eggs) and a significant correlation was found between male size and the number of eggs in the marsupium $(n=$ 58, $r=0.554, P<0 \cdot 01$ ) (data from males that were already pregnant when collected) (Fig. 1).

The courtship and mating ritual, unlike the otherwise cryptic nature of the species, was marked by rather conspicuous behaviours, namely several movements in the water column, above the vegetation canopy. Mating occurred

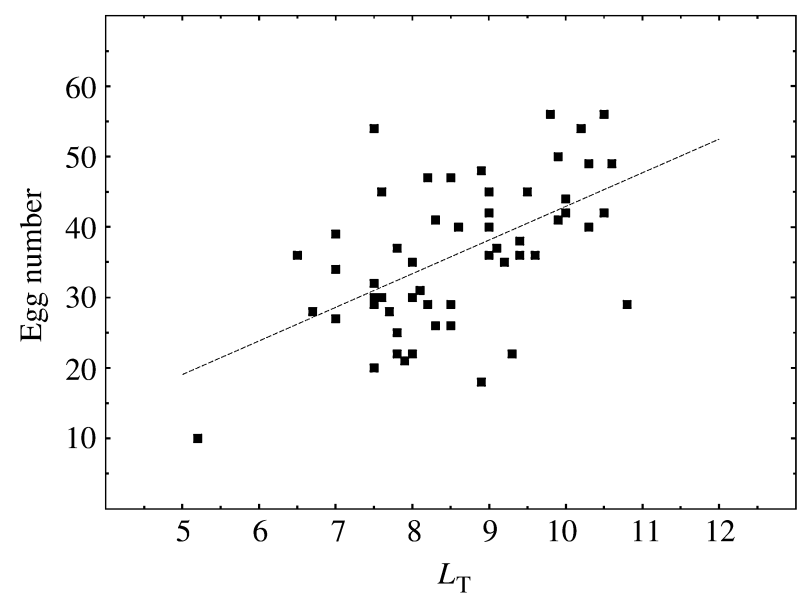

FIG. 1. Relationship between male total length and the number of eggs carried. The curve was fitted by $y=4 \cdot 7742 x-4 \cdot 8064$. 
throughout the day and was preceded by an increase in basal activity levels together with numerous interactions among the fish. This increase was specially pronounced in females that usually initiated displays, mainly consisting of vertical swimming movements in the water column. The onset of courtship was also marked by easily perceivable morphological alterations on both sexes. Females showed an expanded genital papilla (a small, fleshy area near the anus, from which the eggs were released) and a more contrasted colouration, especially in the trunk (Fig. 2). Males presented swollen pouch folds, particularly in the vicinity of the genital area.

The reproductive ritual of $S$. abaster consisted of three distinct phases, marked by prominent behavioural changes that can be summarized as follows. Initial courtship [Fig. 3(a), (b), (c)]: the first stage of the courtship ritual was characterized by mutual flickering movements that affected the entire body rather than just the anterior section of the fish, as observed in Nerophis lumbriciformis (Jenyns) (Monteiro et al., 2002). It consisted mainly of rapid and vigorous bends moving along the main axis of the body. Both females and males approached the opposite sex, without either of the sexes showing greater initiative $(n=17$ courtship trials, male: nine initiatives, female: eight initiatives, $P>$ 0.05). Females tended to flicker first $\left(\chi^{2}, n=17\right.$ courtship trials, male: 3, female: $14, P<0.05)$, but not more often $\left(\chi^{2}, n=142\right.$ flickering movements observed in 17 courtship trials, male: 69 , female: $73, P>0.05)$. After the first flicker, if the opposite sex flickered in response, both individuals performed rapid side-by-side vibrations while swimming through the aquarium in a more or less parallel position (73\% of trials). Females tended to lead these short movements $\left(\chi^{2}, n=34\right.$ leads in 17 courtship trials; male: nine leads, female: 25 leads; $P<0 \cdot 01)$ that spanned over mean \pm s.D. $50 \cdot 2 \pm 21 \cdot 1 \mathrm{~cm}$, and generally occurred only a few cm above the substratum (mean \pm S.D. $8 \cdot 8 \pm 3 \cdot 8 \mathrm{~cm}$ ) but were sometimes ( $n=5$ courtship trials) interrupted by more pronounced elevations in the water column (up to $35 \mathrm{~cm}$ ). During this stage, both males and females approached its partner, both from below $(35 \%)$ and above $(65 \%)$, and touched either with the tails $(57 \%)$ or flanks $(43 \%)$ [Fig. 3(b), (c)]. The flickering behaviour continued, with increased frequency, and the female's genital papilla became clearly visible. The relative length of time of this phase, from the first flicker to the beginning of spawning, was highly variable among observed pairs $(n=10$, range: $1-18$ min, mean \pm s.D. $=8 \pm 7$ $\min$ ), due to a possible variability in the predisposition to mate, but mainly due to disruptions caused by other individuals (especially females; 15 out of 16 observed disruptions) that tended to place their bodies between the courting
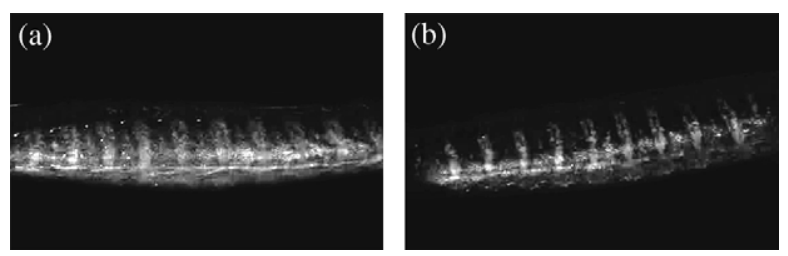

FIG. 2. Female 'amplifiable ornament' (a) in isolation and (b) interacting with another female (increasing contrast in the striped pattern). 

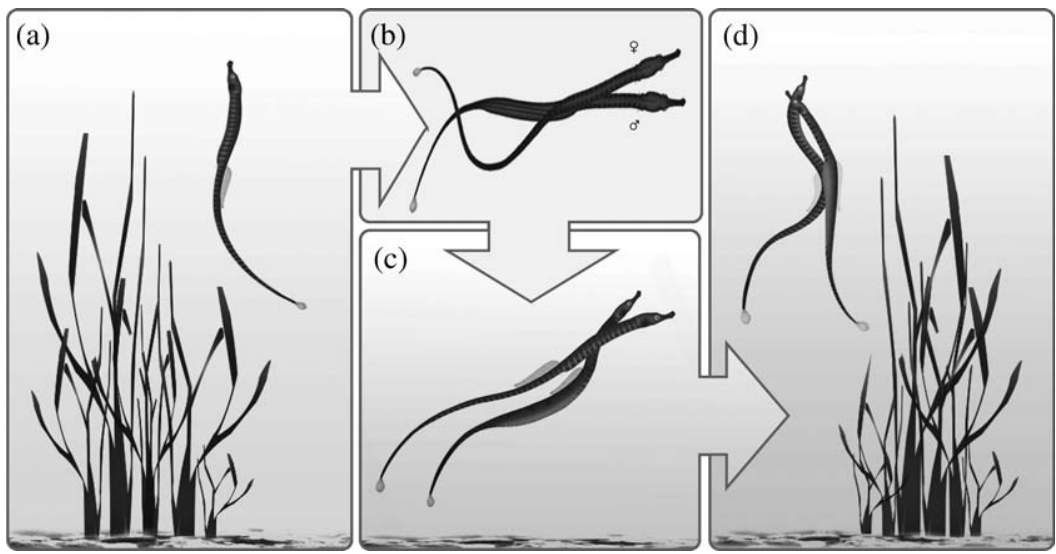

FIG. 3. Overview of the main behavioural stages of courtship and mating: (a) vertical swimming, (b) crossing, (c) parallel swimming and (d) spawning.

pair. Spawning [Fig. 3(d)]: after the initial courtship phase, the female placed its distended genital papilla in the anterior area of the male's marsupium, whose pouch folds were separated and visibly swollen. As soon as the genital areas were in close contact, the mating pair slowly ascended in the water column (up to $45 \mathrm{~cm}$ ), vigorously vibrating the dorsal fin and usually performing a few rotations (11 out of 17 documented matings). During this spawning phase, that lasted, mean \pm S.D. $16 \pm 6 \mathrm{~s}(n=17$, range: $6-25 \mathrm{~s})$, the female rapidly transferred a few eggs into the male's brood pouch $(n=14$, mean \pm S.D. $=$ $17 \pm 6$ eggs, range: 4-30 eggs). Falling eggs, outside the marsupium, were visible in $24 \%$ of the observed transfers. Swaying: immediately after egg transfer, the female swam directly into the substratum while the male continued to swim in the water column performing quick and violent body contractions. This behaviour was probably responsible for the packing of the eggs in the posterior end of the marsupium. Even though sperm ejaculation was not directly visible, fertilization probably occurs inside the marsupium, immediately after egg transfer (Kvarnemo \& Simmons, 2004). At the end of this phase $(n=17$, mean \pm S.D. $=26 \cdot 0 \pm 3 \cdot 0 \mathrm{~s}$, range: $21-31 \mathrm{~s})$, the male slowly descended to the substratum where it stayed motionless (mean \pm S.D. 95.0 $\pm 85 \cdot 4 \mathrm{~s}$ ), sometimes assuming an S-like position (seven in 17 matings), as also observed in Syngnathus acus L. and Syngnathus typhle L. (Vincent et al., 1995).

After a variable latency period (mean \pm s.D. $=15 \cdot 0 \pm 6 \cdot 4 \mathrm{~min})$, a new courtship ritual might occur. In aquaria, some pairs (three in eight) were observed to spawn up to three consecutive times and, interestingly, one female was observed spawning with three different males within $<30 \mathrm{~min}$. Even though the number of mating pairs was low, a significant correlation was observed between male and female size $(n=17, r=0.920, P<0.01)$. In the case of courting pairs that did not achieve copulation, a non-significant correlation was observed ( $n=11, r=0.042, P<0.05)$.

The general pattern of courtship behaviour seems reasonably conserved within the family, basically consisting of three main phases: prespawning, 
egg transfer and postspawning, with fertilization probably occurring during or shortly after egg transfer from the female to the male's incubating surface (Fiedler, 1954; Vincent et al., 1995; Masonjones \& Lewis, 1996; Matsumoto \& Yanagisawa, 2001; Monteiro et al., 2002; Berglund \& Rosenqvist, 2003). Although behaviour analysis was based solely on diurnal observations, the courtship ritual observed in S. abaster falls within this general framework. This general pattern found in diverse genera representing distinct phylogenetic lineages (Wilson et al., 2003) seems to be secondarily modified in some species that live in habitats where displays in the water column are probably disadvantageous. Indeed, a comparison of two species of the genus Nerophis seems informative in this respect: Nerophis ophidion (L.) spawns in relative calm waters, rising up before, during and after egg transfer (Fiedler, 1954), whereas $N$. lumbriciformis, which spawns in the rock intertidal, shows a complete absence of vertical displays (Monteiro et al., 2002). A similar evolutionary reduction of courtship displays in the water column was also documented by Almada \& Santos (1995) for blenniids that spawn in habitats subjected to strong wave action.

Another widespread phenomenon seems to be the occurrence of disruptions during the first phase of the courtship ritual. During the aquarium observations, females were observed approaching the courting pair and beginning to actively flicker or merely following the pair in a parallel motion, a behaviour that might be viewed as a form of competition (Matsumoto \& Yanagisawa, 2001). Curiously, the intruding female was usually unable to mate with the courting male. Only in one occasion was the opposite observed. Nevertheless, these disrupting females were far more successful in redirecting the other female's attention, causing the apparent end of the ongoing courtship display. Similar observations have been described for Corythoichthys haematopterus (Bleeker) (Matsumoto \& Yanagisawa, 2001), Syngnathus schlegeli Kaup (Watanabe et al., 2000) and N. lumbriciformis (Monteiro et al., 2002), suggesting that the still poorly understood femalefemale interactions, that greatly vary among syngnathid genera, play an important role in the competition for access to mates (Berglund \& Rosenqvist, 2003). As in N. ophidion and S. typhle (Berglund \& Rosenqvist, 2003), the apparent absence of overtly aggressive interactions among $S$. abaster females suggests that female dominance occurs mainly through sexual signalling, namely a more contrasted colouration in the trunk (see Fig. 2). This ornamentation seems to be an amplification of the normal colouration, similar to what has been described both for S. typhle (Berglund \& Rosenqvist, 2003) and $N$. lumbriciformis (Monteiro et al., 2002), a phenomenon that might be directly involved not only in male attraction but also in female-female interactions.

The observed sexual dimorphism in size (females being larger than males) and behaviour suggest sex-role reversion in the sampled population of S. abaster (at least under an even sex ratio), a phenomena already documented for several other syngnathids (Kornienko, 2001; Monteiro et al., 2002; Berglund \& Rosenqvist, 2003) as well as other fish families [Kuwamura, 1985 (Apogonidae); Balshine-Earn \& McAndrew, 1995 (Cichlidae); Swenson, 1997 (Gobiidae)]. 
Recently, several hypotheses have been presented on the evolution of sex-role reversal in syngnathids, superimposing behavioural information on phylogenetic data (Wilson et al., 2003). Still far from a definitive result, genetic evidence suggests a pattern still difficult to analyse within syngnathids, with sex-role reversal appearing to be largely independent of pouch complexity (Wilson et al., 2003). These difficulties may well arise from two distinct factors: 1) observations do not take into account the natural variation in the degree of sex-role reversal that might exist in geographically distinct populations within a species. Factors such as the operational sex ratio (Vincent et al., 1994), mate quality (Berglund \& Rosenqvist, 2003), differences between sexes in the relative 'time in' (availability to mate) $v$. 'time out' period (Masonjones $\&$ Lewis, 2000), as well as predation, breeding resources and mate rate encounters are thought to locally modulate the final expression of the sex-role reversal, not only in syngnathids (Almada et al., 1995; Forsgren et al., 2004). For example, S. schlegeli, from Vostok and Amurskii bays, is described as presenting conventional sex roles by Kornienko (2001), while Watanabe et al. (2000) present data suggesting that the same species, from Otsuchi and Funakoshi Bays, is a sexually reversed syngnathid; 2) observations largely depend on a binary definition of sex-role reversal (reversed $v$. conventional). Although S. abaster proved to be sex-role reversed, there is a considerable difference in the magnitude of some measured variables, such as the role of the male during courtship ritual, when compared with other Western European syngnathids, also reported as sex-role reversed species (Kornienko, 2001; Monteiro et al., 2002; Berglund \& Rosenqvist, 2003). Syngnathus abaster males have a more active role in courtship, flickering and sometimes approaching females, when compared to $N$. lumbriciformis (Monteiro et al., 2002), a species where males are much more passive. Moreover, sexual dimorphism is more pronounced in $N$. lumbriciformis (Monteiro et al., 2002) and $N$. ophidion (Berglund \& Rosenqvist, 2003), with differences in colouration among the sexes being observed not only in the middle region of the body (amplifiable ornament display) but also in the head of the females. Nerophis ophidion females present bright blue colour markings along the sides of the head while $N$. lumbriciformis females exhibit an intricate pattern of bright facial spots (Monteiro et al., 2005a). A moderate sex-role reversion has also been reported for the tidewater goby, Eucyclogobius newberryi (Girard), where females display a secondary sexual trait and compete for mates more intensely than do males. Nevertheless, males also engage in intrasexual competition for territories and sometimes also initiate courtship (Swenson, 1997).

More detailed information on syngnathid courtship and mating patterns, encompassing population heterogeneity and what seems to be the clinal nature of reproductive behaviours, would certainly allow for a more conclusive phylogenetic analyses of the evolution of the reproduction within the Syngnathidae family.

Although genetic data are lacking and observations reported in this paper are from captive fish, available evidence suggests that this pipefish is polygynandrous since females were observed mating with different males, sometimes within a very short time period, and males were also observed receiving 


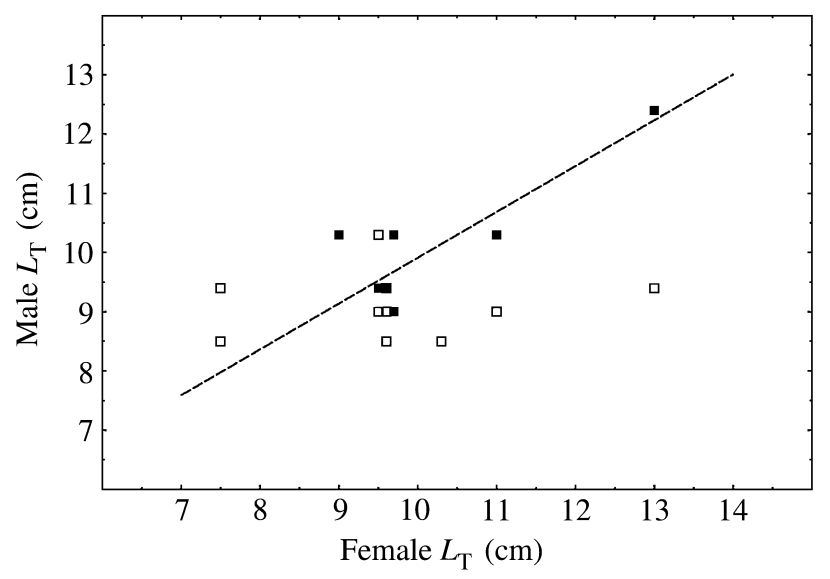

FIG. 4. Male and female total length relationships in successful $(\boldsymbol{\square} ; y=0 \cdot 7774 x+2 \cdot 16)$ and unsuccessful ( $\square$; NS) Syngnathus abaster mating pairs.

eggs from distinct females. In general, more polyandrous species show greater degrees of sexual dimorphism, as a result of an increased intensity in sexual selection (Avise et al., 2002). Polygynandrous mating systems, however, have been reported for two sex-role reversed species with intermediate degrees of sexual dimorphism [S. typhle and Syngnathus floridae (Jordan \& Gilbert) Jones \& Avise, 2001], as well as for the strongly sexual dimorphic sex-role reversed N. lumbriciformis (Monteiro et al., 2006), possibly suggesting a more elaborated pattern than the generally accepted continuum sexual selection-mating system (Avise et al., 2002).

Clutch size of pregnant $S$. abaster males (10-64 eggs) are at the lower end of the range reported for other syngnathids, with Hippocampus reidi Ginsburg and Hippocampus erectus Perry presenting the largest reported clutch size (Monteiro et al., 2005b).

The observed significant correlation between male size and egg number may reflect a $L_{\mathrm{T}}$-dependent reproductive fitness (Fig. 1), with larger males accommodating more offspring, as also reported for S. typhle (Berglund \& Rosenqvist, 2003) and S. schlegeli (Watanabe \& Watanabe, 2002). Accordingly, $L_{\mathrm{T}}$ seems also to affect mate choice, as suggested by the observed significant correlation between male and female size $\left(n=17\right.$ successful mating pairs, $r^{2}=$ 0.846; Fig. 4). Courting pairs with more asymmetrical $L_{\mathrm{T}}$ seemed less successful in achieving mating $\left(n=11\right.$ unsuccessful mating pairs, $r^{2}=0.002$, $P>0 \cdot 05)$.

We would like to thank the anonymous referees for their criticism and suggestions as well as everyone who helped during the fieldwork, especially A. Silva, A. Jorge and P. Correia. V. Almada's participation was partially funded by Programa Plurianual de Apoio às Unidades de Investigação. N. Monteiro's participation was funded by Fundação para a Ciência e a Tecnologia. K. Silva's participation was funded by Fundação para a Ciência e a Tecnologia (FCT-SFRH/BD/13171/2003). 


\section{References}

Almada, V. C. \& Oliveira, R. F. (1997). Sobre o uso de estatística de simulação em estudos de comportamento. Análise Psicológica 1, 97-109.

Almada, V. C. \& Santos, A. J. (1995). Parental care in the rocky intertidal: a case study of adaptation and exaptation in Mediterranean and Atlantic blennies. Reviews in Fish Biology and Fisheries 5, 23-37.

Almada, V. C., Gonçalves, E. J., Oliveira, R. F. \& Santos, A. J. (1995). Courting females: ecological constraints affect sex roles in a natural population of the blenniid fish Salaria pavo. Animal Behaviour 49, 1125-1127.

Avise, J. C., Jones, A. G., Walker, D. \& DeWoody, J. A. (2002). Genetic mating systems and reproductive natural histories of fishes: lessons for ecology and evolution. Annual Review of Genetics 36, 19-45.

Balshine-Earn, S. \& McAndrew, B. J. (1995). Sex-role reversal in the black-chinned tilapia, Sarotherodon melanotheron (Rupel) (Cichlidae). Behaviour 132, 861-874.

Berglund, A. \& Rosenqvist, G. (2003). Sex role reversal in pipefish. Advances in the Study of Behaviour 32, 131-167.

Cakic, P., Lenhardt, M., Mickovic, D., Sekulic, N. \& Budakov, L. J. (2002). Biometric analysis of Syngnathus abaster populations. Journal of Fish Biology 60, $1562-1569$.

Carcupino, M., Baldacci, A., Mazzini, M. \& Franzoi, M. (1997). Morphological organization of the male brood pouch epithelium of Syngnathus abaster Risso (Teleostea, Syngnathidae) before, during, and after egg incubation. Tissue Cell 29, 21-30.

Dawson, C. E. (1986). Syngnathidae. In Fishes of the North-eastern Atlantic and the Mediterranean (Whitehead, P. J. P., Bauchot, M. L., Hureau, J. C., Nielsen, J. \& Tortonese, E., eds), pp. 628-639. Paris: Unesco.

Fiedler, K. (1954). Vergleichende verhaltensstudien an seenadeln, schlangennadeln und seepferdchen (Syngnathidae). Zeitschrift Tierpsychologie 11, 358-416.

Forsgren, E., Amundsen, T., Borg, A. A. \& Bjelvenmark, J. (2004). Unusually dynamic sex roles in a fish. Nature 429, 551-554.

Herald, E. S. (1959). From pipefish to seahorse - a study of phylogenetic relationships. Proceedings of the Californian Academy of Sciences 29, 465-473.

Jones, A. G. \& Avise, J. C. (2001). Mating systems and sexual selection in male-pregnant pipefishes and seahorses: insights from microsatellite-based studies of maternity. The Journal of Heredity 92, 150-158.

Johnstone, R. A. (1995). Sexual selection, honest advertisement and the handicap principle: reviewing the evidence. Biological Reviews 70, 1-65.

Kornienko, E. S. (2001). The spawning behaviour of the pipefish Syngnathus acusimilis. Russian Journal of Marine Biology 27, 54-57.

Kuwamura, T. (1985). Social and reproductive behavior of three mouthbrooding cardinalfishes, Apogon doederleini, A. niger and A. notatus. Environmental Biology of Fishes 13, 17-24.

Kvarnemo, C. \& Simmons, L. W. (2004). Testes investment and spawning mode in pipefishes and seahorses (Syngnathidae). Biological Journal of the Linnean Society 83, 369-376.

Martin, P. \& Bateson, P. (1993). Measuring Behaviour: an Introductory Guide, 20th edn. Cambridge: Cambridge University Press.

Masonjones, H. D. \& Lewis, S. M. (1996). Courtship behavior in the dwarf seahorse, Hippocampus zosterae. Copeia 1996, 634-640.

Masonjones, H. D. \& Lewis, S. M. (2000). Differences in potential reproductive rates of male and female seahorses related to courtship roles. Animal Behaviour 59, 11-20.

Matsumoto, K. \& Yanagisawa, Y. (2001). Monogamy and sex role reversal in the pipefish Corythoichthys haematopterus. Animal Behaviour 61, 163-170.

Monteiro, N. M., Vieira, N. M. \& Almada, V. C. (2001). The breeding ecology of the pipefish Nerophis lumbriciformis and its relation to latitude and water temperature. Acta Ethologica 81, 1031-1033. 
Monteiro, N. M., Vieira, N. M. \& Almada, V. C. (2002). The courtship behaviour of the pipefish Nerophis lumbriciformis: reflections of and adaptation to intertidal life. Acta Ethologica 4, 109-111.

Monteiro, N. M., Vieira, N. M. \& Almada, V. C. (2005a). Homing behaviour and individual identification of the pipefish Nerophis lumbriciformis (Pisces: Syngnathidae): a true intertidal resident? Estuarine, Coastal and Shelf Science 63, 93-99.

Monteiro, N. M., Almada, V. C. \& Vieira, N. M. (2005b). Implications of different brood pouch structures in syngnathid reproduction. Journal of the Marine Biological Association of the United Kingdom 85, 1235-1241.

Monteiro, N. M., Berglund, A., Vieira, N. M. \& Almada, V. C. (2006). Reproductive migrations of the sex role reversed pipefish Nerophis lumbriciformis (Pisces: Syngnathidae). Journal of Fish Biology 69, 66-74.

Ridley, M. (1978). Parental care. Animal Behaviour 26, 904-932.

Silva, K., Monteiro, N. M., Vieira, M. N. \& Almada, V. C. (2006). Early life history of Syngnathus abaster (Pisces: Syngnathidae). Journal of Fish Biology 68, 80-86.

Swenson, R. O. (1997). Sex-role reversal in the tidewater goby, Eucyclogobius newberryi. Environmental Biology of Fishes 50, 27-40.

Tomasini, J. A., Quignard, J. P., Capapé, C. \& Bouchereau, J. L. (1991). Facteurs du succés reproductif de Syngnathus abaster Risso, 1826 (Pisces, Telestei, Syngnathidade) em milieu lagunaire mediterranéen (lagune de Mauguio, France). Acta Oecologica 12, 331-355.

Trivers, R. L. (1972). Parental investment and sexual selection. In Sexual Selection and the Descent of Man (Campbell, B., ed.), pp. 136-179. Chicago, IL: Aldine-Atherton.

Vincent, A., Ahnesjo, I. \& Berglund, A. (1994). Operational sex rations and behavioural sex differences in a pipefish population. Behavioural Ecology and Sociobiology 34, 435-442.

Vincent, A., Berglund, A. \& Ahnesjo, I. (1995). Reproductive ecology of five pipefish species in one eelgrass meadow. Environmental Biology of Fishes 44, 347-361.

Watanabe, S. \& Watanabe, Y. (2002). Relationship between male size and newborn size in the seaweed pipefish, Syngnathus schlegeli. Environmental Biology of Fishes 65, 319-325.

Watanabe, S., Hara, M. \& Watanabe, Y. (2000). Male internal fertilization and introsperm-like sperm of the seaweed pipefish Syngnathus schlegeli. Zoological Science 17, 759-767.

Wilson, A. B., Ahnesjo, I., Vincent, A. C. J. \& Meyer, A. (2003). The dynamics of male brooding, mating patterns, and sex roles in pipefishes and seahorses (Family Syngnathidae). Evolution 57, 1374-1386.

Zar, J. H. (1984). Biostatistical Analysis. Upper Saddle River, NJ: Prentice-Hall. 